\title{
Total aortic arch replacement under intermittent pressure-augmented retrograde cerebral perfusion
}

\author{
Hiroshi Kubota ${ }^{1 *}$, Kunihiko Tonari $^{1}$, Hidehito Endo ${ }^{1}$, Hiroshi Tsuchiya' ${ }^{1}$ Hideaki Yoshino ${ }^{2}$, Kenichi Sudo
}

\begin{abstract}
Kitahori, Kawata, Takamoto et al. described the effectiveness of a novel protocol for retrograde cerebral perfusion that included intermittent pressure augmentation for brain protection in a canine model. Based on their report, we applied this novel technique clinically. Although the duration of circulatory arrest with retrograde cerebral perfusion was long, the patient recovered consciousness soon after the operation and had no neurological deficit. Nearinfrared oximetry showed recovery of intracranial blood oxygen saturation every time the pressure was augmented.
\end{abstract}

\section{Background}

To prolong the safe limits of conventional retrograde cerebral perfusion (RCP), Kitahori, Kawata, Takamoto et al. assessed a novel protocol, intermittent pressureaugmented retrograde cerebral perfusion (IPA-RCP), in a canine model [1-3]. This new protocol was clinically applied to a 51 year-old-male with a diagnosis of acute aortic dissection. Near infrared oximetry showed recovery of intracranial blood oxygen saturation during the pressure augmentation. Although duration of RCP was long, the patient recovered consciousness $30 \mathrm{~min}$ after the operation free of any neurological deficit after total arch replacement.

\section{Case presentation}

On July 24, 2006, a 51 year-old-male with a diagnosis of acute aortic dissection (DeBakey I, Stanford A) was transferred to our hospital from a nearby hospital, and emergency operation was performed the same day. The pericardium was opened through a median sternotomy and a cardiopulmonary bypass was established by cannulations the inferior and superior venae cavae and the right femoral artery. Circulatory arrest with retrograde cerebral perfusion was commenced when the patient's tympanic temperature reached to $18.0^{\circ} \mathrm{C}$. A large longitudinal intimal tear was present in the greater curvature

\footnotetext{
* Correspondence: kub@ks.kyorin-u.ac.jp

'Department of Cardiovascular Surgery, Kyorin University, Tokyo, Japan

Full list of author information is available at the end of the article
}

of the aortic arch, and it ended just proximal to the left subclavian artery. The aorta was transected between the left common carotid artery and the left subclavian artery. The aorta was reinforced with two Teflon felt strips, and a four-branch 24-mm graft was anastomosed. After anastomosis of the left common carotid artery, the graft was clamped, and antegrade perfusion via a side branch and rewarming were started. The brachiocephalic artery was then anastomosed and perfused. Finally, the proximal anastomosis was performed, and the aortic clamp was released. Weaning from the cardiopulmonary bypass was achieved smoothly.

\section{Retrograde cerebral perfusion}

Conventional retrograde cerebral perfusion (RCP) with $15 \mathrm{mmHg}$ of superior vena cava pressure was performed first, and $30 \mathrm{~min}$ later, when the anesthesiologist alert that near-infrared oximetry showed a low value under $50 \%$, we converted to the intermittent pressure augmented retrograde cerebral perfusion (IPA-RCP) method with superior vena cava pressure increased to 45 $\mathrm{mmHg}$. The intervals and durations of the augmentations were irregular, because when the backflow from the cervical branch disturbed the anastomosis, the pressure decreased expediently. The maximum duration of augmentation was limited to $30 \mathrm{sec}$. The circulatory arrest time, conventional RCP time, IPA-RCP time were $85 \mathrm{~min}, 30 \mathrm{~min}$, and $55 \mathrm{~min}$, respectively, and a total of 10 augmentations were performed. Intracranial regional 
oxygen saturation $\left(\mathrm{rSO}_{2}\right)$ was measured with a TOS-96 brain oximeter (TOSTEC Co., Ltd. Tokyo, Japan).

\section{Results}

Prior to the anesthesia, the $\mathrm{rSO}_{2}$ was $61 \%$ (Left) and $60 \%$ (Right). At the beginning of the cardiopulmonary bypass, the $\mathrm{rSO}_{2}$ was $55 \%$ (Left) and $56 \%$ (Right). At profound hypothermia, the $\mathrm{rSO}_{2}$ was $64 \%$ (Left) and $63 \%$ (Right), it gradually decreased to $49 \%$ (Left) and $50 \%$ (Right). After commencing the IPA-RCP, the rSO2 rose to around $60 \%$ at every augmentation, but it decreased when the augmentation ceased. Just after the resuming antegrade perfusion via a side branch of the graft, the $\mathrm{rSO}_{2}$ decreased to $40 \%$, then recovered smoothly (Figure 1). The $\mathrm{rSO}_{2}$ on the right side recovered in a stepwise manner. The patient recovered consciousness $30 \mathrm{~min}$ after the operation free of any neurological deficit and the postoperative course was uneventful.

\section{Conclusions}

RCP by augmentation of CVP to 15 to $20 \mathrm{mmHg}$ is routinely used in our institute for the additional brain protection during deep hypothermic circulatory arrest because much evidence has been accumulated to suggest an increased risk of perfusion-induced brain injury associated with RCP, especially when continuously high RCP pressures are used [4]. However, there is a safety limit of the deep hypothermic circulatory arrest duration because it cannot open all intracranial vessels but partially. To overcome this drawback, Kitahori, Kawata, Takamoto et al. developed a new intermittent pressure augmentation method in which CVP is intermittently increased to $45 \mathrm{mmHg}$ [1-3]. They used a canine model, and showed that the retinal vessels were effectively dilated at an augmented pressure of $45 \mathrm{~mm} \mathrm{Hg}$ (arteries, $107 \%+3 \%$ of control veins, $114 \%+3 \%$ of control), whereas when antegrade selective cerebral perfusion was used, the retinal vessels were smaller than the corresponding preoperative vessels. They concluded that the intermittent pressure augmentation allows an adequate blood supply without injuring the brain and provides adequate neuroprotection equivalent to that provided by antegrade cerebral perfusion. In the canine model, they administered the RCP through the maxillary vein to overcome the drawbacks of jugular vein valves to reach directly the cranial veins. In the majority of humans, as de Brux et al. described, the jugular vein had competent valves and it is hypothesized that the RCP gains the brain through a collateral network of veins (azygos, intercostal, medullary and vertebral veins). The usefulness of higher perfusion pressure could be either to distend the valves or more probably to increase the pressure in the collateral vein network to improve cerebral oxygenation [5]. Thus, the clinical effectiveness of the IPA-RCP through a cannulae inserted to the SVC is unknown field. We examined the effect of the IPA-RCP by measuring $\mathrm{rSO}_{2}$ which represents the brain blood

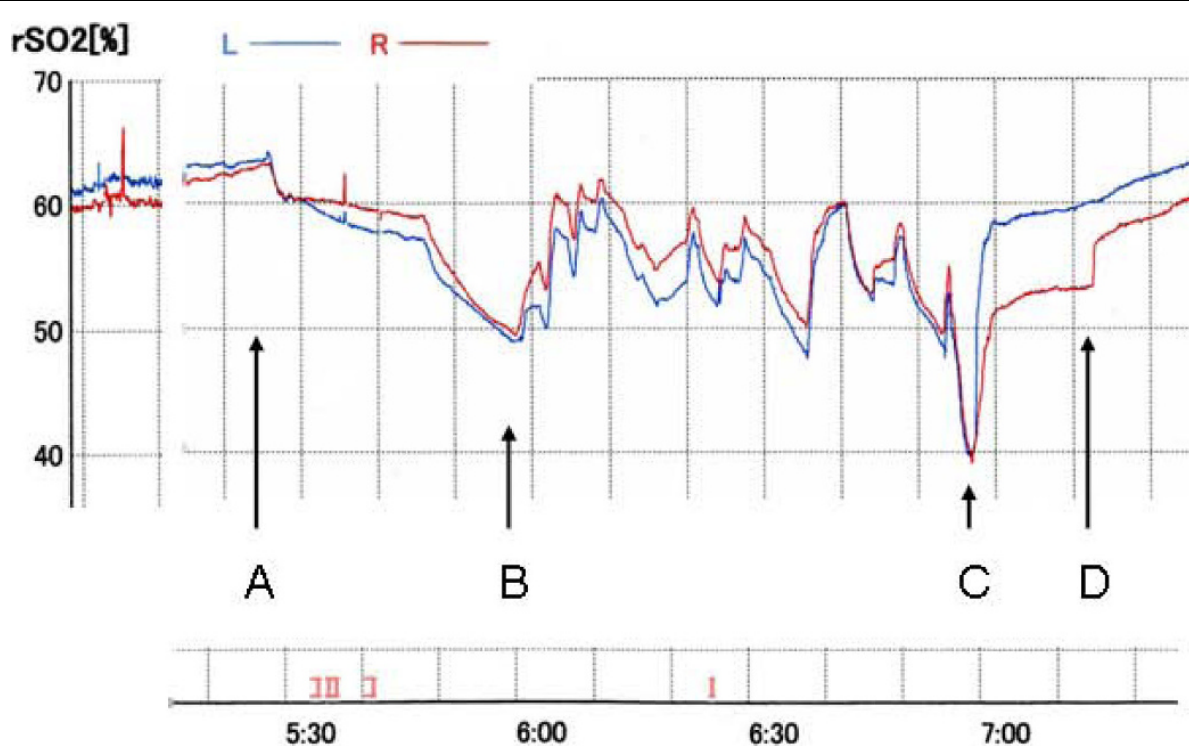

Figure $1 \mathrm{rSO}_{2}$ during deep hypothermic circulatory arrest. L: left $\mathrm{rSO}_{2}$, R: right $\mathrm{rSO}_{2}$. Initial 30 min of conventional retrograde cerebral perfusion (RCP), $\mathrm{rSO}_{2}$ gradually declined. When intermittent-pressure-augmented $(45 \mathrm{mmHg}$ ) retrograde cerebral perfusion (IPA-RCP) was induced, $\mathrm{rSO}_{2}$ rose. The maximum duration of pressure augmentation was limited to $30 \mathrm{sec}$. A total of 10 augmentations at irregular intervals were tried. A. Start of deep hypothermic circulatory arrest and conventional RCP. B. Start of IPA-RCP. C. Final dip: Start of the antegrade perfusion to the left common carotid artery, and the left subclavian artery via graft branch. D. Start of antegrade perfusion via the brachiocephalic artery. 
perfusion. Although only the anterior part of the brain $\mathrm{rSO}_{2}$ is assessed by a TOS-96 brain oximeter, because most attenuation of near-infrared light in human cerebral tissues is due to absorption by deoxyhemoglobin and oxyhemoglobin, brain tissue is suitable for determination of $\mathrm{rSO} 2$. Only determination of $\mathrm{rSO} 2$ is an easily available method to assess the real-time adequacy of cerebral perfusion during deep hypothermic timerestricted aortic arch surgery [6].

At first, we planned to perform the operation on our patient using conventional RCP. However, because the rSO2 declined to $49 \%$, the duration of circulatory arrest time was expected to exceed $60 \mathrm{~min}$ due to the fragile aortic wall to reinforce and deep distal anastomosis, we applied the intermittent pressure augmentation technique for the first time. According to the original report, the central venous pressure was controlled at $15 \mathrm{~mm}$ $\mathrm{Hg}$ and it was augmented to $45 \mathrm{~mm} \mathrm{Hg}$ quickly and then decreased again to the baseline level of $15 \mathrm{mmHg}$ as soon as it reached $45 \mathrm{~mm} \mathrm{Hg}$ every 30 seconds. However, the same protocol is difficult to apply clinically because backflow from the three arch vessels increased and disturbed the anastomosis when CVP was augmented. CVP was decreased to $15 \mathrm{mmHg}$ expediently. Although the optimal duration of pressure augmentation during deep hypothermic circulatory arrest in clinical settings is unknown, to prevent the brain edema, the maximum duration of pressure augmentation that we set was $30 \mathrm{sec}$.

Along with every pressure augmentation, rSO2 showed immediate recovery up to $60 \%$ and it decreased when the augmentation ceased. The essential effect of IPA-RCP may not only be a temporary increase in $\mathrm{rSO} 2$ but elevation of the declining curve during RCP. Our preliminary randomized comparative study in clinical aortic arch replacement cases of IPA-RCP $(n=10)$ and standard RCP $(n=10)$ showed that the interval from the end of the operation to full awakeness of the IPA$\mathrm{RCP}$ group was $85 \pm 64 \mathrm{~min}$. in contrast with $310 \pm 282$ min. in RCP group $(\mathrm{p}<0.05)$ accompanying with the $\mathrm{rSO} 2$ decline ratio $60 \mathrm{~min}$ after the initiation of the IPA-RCP group was $13.1 \pm 3.7 \%$ in contrast with $24.5 \pm$ $13.1 \%$ in RCP group $(\mathrm{p}<0.05)$. There was no significant difference of the used amount of the anesthetic agent. It may support the "bottom raising effect" of this new protocol.

Just after the resumption of antegrade perfusion, the $\mathrm{rSO}_{2}$ decreased to $40 \%$, but then recovered smoothly. We named this phenomenon the "final dip". When we use RCP, the final dip always appears just after the resumption of antegrade perfusion. This phenomenon may represent wash out of deoxygenated blood that remained and did not circulate in the brain despite the performance of retrograde cerebral perfusion. The stepwise recovery of the $\mathrm{rSO}_{2}$ of the right side may mean that the resumption of antegrade perfusion via the left arch branches was insufficient to wash out the remaining blood in our patient. In conclusion, this novel protocol may have some advantages over conventional RCP. Because it is difficult to verify the efficacy of IPARCP by quantitative analysis, accumulation and analysis of data e.g. measurement of the concentration of Tau proteins in the CSF, comparison of the pre- and postoperative cognitive function, measurement of the diameters of the retinal vessels during IPA-RCP may demonstrate the advantages of this new method of brain protection [7].

\section{Acknowledgements}

We would like to gratefully acknowledge the outstanding original idea of the IPA-RCP protocol, laboratory investigation, and cooperation given to us by all the cardiac surgeons at the Mitsui Memorial Hospital: S Takamaoto, T Miyairi, Columbia University Medical Center: H Takayama, and Tokyo University Hospital: M Kawata, T Taketani, K Kitahori, K Nawata, T Morota, N Motomura, M Ono.

\section{Author details}

'Department of Cardiovascular Surgery, Kyorin University, Tokyo, Japan. ${ }^{2}$ Department of Cardiology, Kyorin University, Tokyo, Japan.

\section{Authors' contributions}

$H K, K T, H E, H T$ conceived of the study, and participated in its design and coordination. HY and SK participated in the sequence alignment. All authors read and approved the final manuscript.

\section{Competing interests}

The authors declare that they have no competing interests.

Received: 7 June 2010 Accepted: 2 November 2010

Published: 2 November 2010

\section{References}

1. Kitahori K, Takamoto S, Takayama H, Suematsu Y, Ono M, Motomura N, Morota T, Takeuchi K: A novel protocol of retrograde cerebral perfusion with intermittent pressure augmentation for brain protection. J Thorac Cardiovasc Surg 2005, 130:363-370.

2. Kawata M, Takamoto S, Kitahori K, Tsukihara H, Morota T, Ono M, Motomura N, Murakami A, Suematsu Y: Intermittent pressure augmentation during retrograde cerebral perfusion under moderate hypothermia provides adequate neuroprotection: An experimental study. J Thorac Cardiovasc Surg 2006, 132:80-88.

3. Kawata M, Sekino M, Takamoto S, Ueno S, Yamaguchi S, Kitahori K, Tsukihara H, Suematsu Y, Ono M, Motomura N, Morota T, Murakami A: Retrograde cerebral perfusion with intermittent pressure augmentation provides adequate neuroprotection: diffusion- and perfusion-weighted magnetic resonance imaging study in an experimental canine model. $J$ Thorac Cardiovasc Surg 2006, 134:933-40.

4. Usui A, Oohara K, Liu TL, Murase M, Tanaka M, Takeuchi E, Abe T: Determination of optimum retrograde cerebral perfusion conditions. $J$ Thorac Cardiovasc Surg 1994, 107:300-8.

5. De Brux JL, Subayi JP, Pegis JD, Pillet J: Retrograde cerebral perfusion: anatomic study of the distribution of blood to the brain. Ann Thorac Surg 1995, 60:1294-8.

6. Ogino H, Ueda Y, Sugita T, Morioka K, Sakakibara Y, Matsubayashi K, Nomoto T: Monitoring of regional cerebral oxygenation by near-infrared spectroscopy during continuous retrograde cerebral perfusion for aortic surgery. Eur J Cardiothorac Surg 1998, 14:415-8.

7. Kubota H, Takamoto S, Yoshino H, Kitahori K, Kawata M, Tonari K, Endo H, Tsuchiya H, Inaba Y, Takahashi Y, Sudo K: Clinical Application of 
Intermittent Pressure-Augmented Retrograde Cerebral Perfusion. Ann Thorac Surg 2010, 90:1340-3.

doi:10.1186/1749-8090-5-97

Cite this article as: Kubota et al: Total aortic arch replacement under

intermittent pressure-augmented retrograde cerebral perfusion. Journal of Cardiothoracic Surgery 2010 5:97.

Submit your next manuscript to BioMed Central and take full advantage of:

- Convenient online submission

- Thorough peer review

- No space constraints or color figure charges

- Immediate publication on acceptance

- Inclusion in PubMed, CAS, Scopus and Google Scholar

- Research which is freely available for redistribution

Submit your manuscript at www.biomedcentral.com/submit
() BioMed Central 